\title{
Reprend-on le travail ou poursuit-on sa vie ?
}

\section{Do we go back to work or do we continue our life ?}

\section{N. Pélicier · P. Bergerot - M.-F. Bacqué}

(C) Springer-Verlag France 2014

Voici un numéro thématique consacré au vécu et aux problématiques de la perte et de la reprise du travail. Les données sociales liées à l'impact de la maladie sont de plus en plus étudiées, prises en compte.

Parmi toutes les composantes de ce qui fait la qualité de vie de nos patients, il nous a semblé indispensable de convier les psycho-oncologues, les équipes soignantes, à s'intéresser à celle plus particulière du travail.

" Je mène une vie quasi normale, je ne me suis pas arrêtée de travailler $\gg . .$. Le repère du travail évoque, pour cette patiente, la permanence des repères de sa vie, malgré la maladie. Il y a là une réalité, pour de nombreux de patients, de la vie professionnelle avec la maladie, avec les traitements.

Le travail n'est pas à replacer dans le seul « après cancer », soit parce que celui-ci reste hypothétique, soit parce que le travail est de l'ordre de la nécessité vitale, de la « ressource », presque à « hauteur» des traitements spécifiques du cancer.

Les associations de patients, les États Généraux de ces dernières décennies nous ont transmis depuis longtemps la difficulté des patients ou de leurs proches, pour préserver, conserver, ou retrouver leur place au travail et leurs capacités.

Contrecoup physique de la maladie, séquelles des traitements, impactent en effet le parcours du « retour au travail ». Mais que dire des retrouvailles avec les collègues, les tâches désormais inadaptées ? Désarroi, amertume, révolte, émaillent le temps de la reprise ou celui du maintien dans l'emploi.

\section{N. Pélicier $(\square)$}

Unité de psycho-oncologie de l'HEGP,

20, rue Leblanc, F-75908 Paris cedex 15, France

e-mail : nicole.pelicier@egp.aphp.fr

\section{P. Bergerot}

Ligue contre le cancer - Collectif interassociatif sur la santé - Centre oncologique de l'estuaire, clinique mutualiste de l'estuaire, 11, boulevard Charpak, F-44606 Saint-Nazaire, France

M.-F. Bacqué

Rédactrice en chef de Psycho-Oncologie

Université de Strasbourg, France
Comment réaliser la feuille de route du Plan cancer 3 et vaincre les inégalités sociales?

Comment préparer les patients au retour à l'emploi en intégrant tous les changements prévisibles ou inattendus?

Nous plaidons tous, dans ce numéro, pour une concertation en amont de la sortie de crise, avec les médecins qui ont à penser, et repenser aussi, leurs choix thérapeutiques en fonction « du devenir au travail ». Mais aussi pour une évaluation avec les familles, les représentants de la société civile, afin que le regard encore posé sur nos patients ne soit pas systématiquement stigmatisant.

Une femme ne dort presque plus, depuis qu'elle a repris son travail à temps partiel, après un cancer et une rechute. Tout pourtant a-t-il été « pensé » ? En amont, elle a eu droit à la « visite de reprise », aux conseils de l'assistante sociale, au certificat du cancérologue. Mais sur place, elle retrouve une entreprise gérée en fonction de marchés et de déficits qui ne concèdent dorénavant plus rien à l'humain ! Dès les premières semaines, la charge du « temps aménagé » a augmenté et débordé le cadre " fixé ». La voilà entrainée vers le stress et l'insomnie. "C'est comme avant », me dit-elle ; avant la maladie.

Or tout a changé, car elle note avec une sorte d'effroi "qu'elle sait que quelque chose a eu lieu »! Après, n'est pas comme avant, et la trace existentielle angoissante laissée par le cancer est ravivée par ce que son travail conserve d'insoutenable. La finitude, dont elle a subitement hérité ne peut se concilier avec un temps bâclé, gâché, par un travail auquel on ne donne plus sa valeur de production personnelle. Il faut bien d'autres changements sociétaux et psychologiques, sans nul doute, pour que l'affrontement ne tourne pas au drame et à la perte.

Écho et résonnance de la perte et de la reprise sont donc ici analysés par différents auteurs, de points de vue divers.

Leur constat revient sur une même nécessité : pendant la maladie, le plus tôt possible, évaluer le contexte social et professionnel d'un patient, connaître son rôle familial (charge de famille, ascendant, descendant), les facteurs de risque psychologiques (antécédents, personnalités, capacités d'adaptation) afin de repérer les sujets fragiles à 
accompagner avec l'aide des travailleurs sociaux, des psychologues et psychiatres.

Après la maladie : favoriser la communication avec le milieu professionnel mais ne pas précipiter le patient dans une reprise contrainte pour « forcer le destin », au-delà de toute réalité possible.

La symbolique du travail, « du faire » est un champ infini à explorer et à élaborer dans une dimension pragmatique et psychothérapeutique.

Les rapports récents, à la fois de l'Observatoire Sociétal du Cancer et l'étude « La vie deux ans après le diagnostic de cancer », éclairent la scène professionnelle de repères chiffrés et incontournables. Ils nous aident à prendre connaissance de l'ampleur des problèmes : avoir et perdre, reprendre mais en combien de temps, à quel prix ? Quels en sont les enjeux ?

Les psycho-oncologues, les équipes soignantes, les tutelles et les réseaux ont donc à se prononcer à travers des prises en charge « améliorées », en vue du travail, un jour possible ou simplement obligé.

Surtout, quelque chose se poursuit et doit se poursuivre dans la responsabilité soignante et sociétale à accompagner les patients dans un retour digne au travail.

" Je suis retourné là où je pensais ne plus jamais aller. Tout s'est remis en place, étrangement vite. J'étais sur un nuage, à mon travail et... ailleurs ».

Ne pas négliger « l'ailleurs » que nous livre l'expérience $\mathrm{du}$ cancer et sa résonnance, nous autorise à demander l'accès aux soins psychiques, dans ce temps souvent en décalage, que constitue la reprise. Sur le chemin du retour, y-aura-t-il des GPS futuristes et inappropriés ou de véritables réseaux de solidarité et de bienveillance ?

Reprend-on le travail ou poursuit-on sa vie ?

Comment, en 2014, entend-on se positionner pour redonner plus de place à la vie qu'au cancer ? 